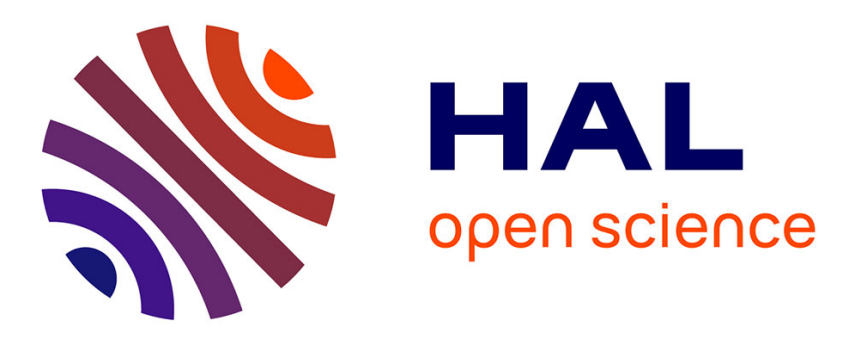

\title{
Lateral Gene Transfer Mechanisms and Pan-genomes in Eukaryotes
}

\author{
Shannon Sibbald, Laura Eme, John Archibald, Andrew Roger
}

\section{To cite this version:}

Shannon Sibbald, Laura Eme, John Archibald, Andrew Roger. Lateral Gene Transfer Mechanisms and Pan-genomes in Eukaryotes. Trends in Parasitology, 2020, 36 (11), pp.927-941. 10.1016/j.pt.2020.07.014 . hal-03368658

\section{HAL Id: hal-03368658 https://hal.science/hal-03368658}

Submitted on 7 Oct 2021

HAL is a multi-disciplinary open access archive for the deposit and dissemination of scientific research documents, whether they are published or not. The documents may come from teaching and research institutions in France or abroad, or from public or private research centers.
L'archive ouverte pluridisciplinaire HAL, est destinée au dépôt et à la diffusion de documents scientifiques de niveau recherche, publiés ou non, émanant des établissements d'enseignement et de recherche français ou étrangers, des laboratoires publics ou privés. 


\section{Lateral gene transfer mechanisms and pan-genomes in eukaryotes}

3 Shannon J. Sibbald ${ }^{1,2 *}$, Laura Eme ${ }^{3}$, John M. Archibald ${ }^{1,2}$ and Andrew J. Roger ${ }^{1,2 *}$

$4{ }^{1}$ Department of Biochemistry \& Molecular Biology, Dalhousie University, Halifax, Nova Scotia,

5 Canada

$6{ }^{2}$ Centre for Comparative Genomics and Evolutionary Bioinformatics, Dalhousie University,

7 Halifax, Nova Scotia, Canada

$8{ }^{3}$ Ecologie Systématique Evolution, CNRS, Université Paris-Sud, Orsay, France

$9 \quad$ *Correspondence: Andrew.Roger@dal.ca (A. J. Roger), Shannon.sibbald@ dal.ca (S. J. Sibbald)

11 Keywords: Lateral gene transfer, horizontal gene transfer, eukaryote genomes; pan-genomes

13 Abstract

14 Lateral gene transfer (LGT) is well known as an important driver of genome evolution in bacte-

15 ria and archaea, but its importance in eukaryote evolution has yet to be fully elucidated. There is

16 now abundant evidence indicating that LGT has played a role in the adaptation of eukaryotes to

17 new environments and conditions, including host-parasite interactions. However, the mecha-

18 nisms and frequency of LGT across the tree of eukaryotes remain poorly understood. Here we

19 review evidence for known and potential mechanisms of LGT into diverse eukaryote lineages

20 with a particular focus on protists, and discuss trends emerging from recently reported examples.

21 We also explore the potential role of LGT in generating 'pan-genomes' in diverse eukaryotic

22 species. 


\section{The role and prevalence of lateral gene transfer in genome evolution}

25 Lateral (or horizontal) gene transfer (LGT or HGT) is an important mechanism by which genetic 26 material is inherited in a non-vertical fashion. In bacteria, DNA is transmitted horizontally by

27 transduction, transformation and conjugation (see Glossary) (e.g., [1]). Although the frequen-

28 cy of LGT varies greatly amongst taxa, these processes are largely responsible for gene content

29 variation between and within prokaryotic species. Indeed, the total gene content of a given spe-

30 cies - the pan-genome - can exceed the number of 'core' genes shared between all strains of

31 that species by an order of magnitude or more; those accessory genes are commonly acquired by

32 LGT (e.g., [2] and references therein). It remains unclear what proportion of the genes found in

33 some but not all strains of a bacterial species are adaptive versus neutral or deleterious $[3,4]$, but

34 there is no doubt that LGT has a major role in prokaryote genome evolution.

By contrast, the prevalence, mechanisms and roles of LGT in eukaryote evolution are

36 poorly understood. Putative LGTs are increasingly reported as the number of comparative ge-

37 nomic analyses of diverse unicellular and multicellular eukaryotes rapidly grows (Box 1). It is

38 generally accepted that LGT likely occurs less frequently in eukaryotes relative to most prokary-

39 otes (e.g., [5]), but this does not mean that it is an unimportant mechanism in eukaryotic genome

40 evolution [6]. Laterally acquired genes can provide eukaryotic organisms with new functions that

41 impact their biology and evolutionary trajectory (e.g., [7]); LGT has been suggested to play a

42 role in the adaptation of eukaryotes to 'extreme' conditions such as high temperature (e.g., [8]),

43 high salt (e.g., [9]), and anaerobiosis (e.g., [10, 11]). LGT also appears to have contributed to the

44 evolution of parasitism (e.g., [12] and references there-in, [13,14]) and in the reversal from a

45 parasitic to a free-living lifestyle (e.g., [15]). 
How do eukaryotes acquire genes? Numerous potential sources of foreign DNA have

47 been suggested (Figure 1) but the evidence supporting their involvement in LGT varies. Some

48 phenomena, like endosymbiotic gene transfer (EGT), are well supported by evidence. Others,

49 such as inter-domain conjugation or natural transformation, have been documented in the lab

50 [16] but their frequencies and relevance in nature is unknown. Regardless of the source, a 'suc-

51 cessful' LGT must involve several steps including: (i) the introduction of foreign DNA (or RNA)

52 into the recipient eukaryotic cell; (ii) translocation of the DNA to the nucleus; and (iii) incorpo-

53 ration of the DNA into one or more chromosomes, either accidentally by host DNA repair and

54 recombination pathways, or by an active integration mechanism. For transferred DNA to be

55 faithfully inherited, it must be stably integrated in all sister chromosomes and, for multicellular

56 eukaryotes, must exist in the germline. To persist over longer time periods, newly acquired DNA

57 must be fixed in the population by neutral drift and/or positive natural selection. If the transferred

58 genes are expressed and provide a function that is useful (e.g., a novel adaptive trait, or compen-

59 sation for the loss of a pre-existing gene), they can be maintained by purifying selection. Other-

60 wise, further mutations may erode these genes into pseudogenes (e.g., [17]) and they will even-

61 tually be lost or become unrecognizable intergenic DNA. Clearly the likelihood of any given

62 LGT event being successful is multifactorial, and a number of factors potentially influence the

63 frequency of LGT in any given species (Box 2).

64 Here we review evidence for several means by which foreign DNA is introduced into

65 eukaryotic cells and potential mechanisms that integrate such DNA into eukaryotic genomes. We

66 also discuss aspects of genome biology that may influence the rate of LGT and discuss its im-

67 portance to adaptive evolution in eukaryotes by acting as a 'catalyst' for the acquisition of niche-

68 specific traits. 


\section{Viruses and their hosts exchange genetic material}

71 Virus-mediated transduction has long been recognized as an integral part of the biology

72 of prokaryotic cells. A growing body of literature supports the notion that viruses can act as vec-

73 tors of LGT from prokaryotes to eukaryotes and between eukaryotes (reviewed in [18]). The

74 nature of viral transmission (horizontal), replication (inside the host cell) and survival strategies

75 (e.g., integration into the host genome) together lead to intimate associations with their host's

76 genetic material, providing ample opportunity for gene exchange. At least some viruses can in-

77 fect a wide range of hosts and/or change hosts (e.g., [19]) and some eukaryotes harbour multiple

78 divergent viruses, each of which may enable gene exchange beyond species boundaries. The

79 virus-mediated export of nuclear DNA in plants was recently documented in real time by Catoni

80 et al. [20], where Beta vulgaris chromosomal DNA integrated into the viral genome of a single-

81 stranded DNA virus during infection was encapsidated into viral particles and spread throughout

82 the plant population via subsequent viral infections. Notably, the B. vulgaris DNA present in the

83 hybrid minicircles was replicated and transcribed in the newly infected host.

84 It is well established that host genes can be integrated into a viral genome and persist,

85 particularly in double stranded DNA (dsDNA) viruses that infect a variety of eukaryotes. In the

86 case of nucleocytoplasmic large DNA viruses, host DNA can make up a substantial portion of

87 the virus' genome (e.g., [21]). A recent study of the giant DNA virus of the green alga

88 Tetraselmis, TetV, identified 110 protein coding genes (17\% of its protein coding genes) that

89 appeared to be acquired from eukaryotes, including many specifically of Viridiplantae origin

90 [21]. At least four of the eukaryotic homologs identified in TetV were novel viral genes that sug-

91 gested recent incorporation of genes from both its host (Tetraselmis) and other unicellular eukar- 
92 yotes that likely allow manipulation of the host fermentation pathways during anaerobic condi-

93 tions. Giant virus genomes are highly mosaic - not only do they acquire genes from their eukar-

94 yotic host(s), they also pick up genes from bacteria and other viruses present within the host cell 95 (e.g., [22]).

96 The fact that genes of host origin exist in viral genomes implies that host DNA is regular-

97 ly introduced into viral genomes with varying consequences on fitness; while some genes are

98 fixed, others presumably persist temporarily over a limited number of replication cycles. Short-

99 lived insertions, however, could still allow the foreign genes to be transferred to a new host. This

100 was shown experimentally by Gilbert et al. [23], who investigated the frequency of gene transfer

101 in 21 baculovirus (AcMNPV) populations infecting two moth species. Around 5\% of AcMNPVs

102 were found to harbor at least one moth-derived DNA sequence at a given time, all of which per-

103 sisted only temporarily. There is nevertheless evidence for a continuous flow of genetic material

104 from the host to AcMNPV and for repeated transfers of the short-lived insertions from the viral

105 genome into the one of the various insects they infect. Host DNA was predominately inserted

106 into viral genomes via transposition. However, there is also evidence for a variety of

107 microhomology-mediated and homology-independent recombination events at double stranded

108 breaks. Whether these recombination events were mediated by host or viral factors, or some

109 combination of the two is currently unknown, but Gilbert et al. [23] speculate that as long as

110 there is a double stranded break, viral and host DNA have the potential to be joined.

111 A large number of viral genomes and genome fragments from a wide range of both retro-

112 viruses and other viruses have been found in eukaryote genomes, including those of multicellular

113 organisms (e.g., [24-26]). In order to replicate, retroviruses become endogenized, i.e., integrated

114 in their hosts genome via an autonomous mechanism using enzymatic machinery they encode. 
115 Beyond retroviruses, it has been suggested that viral DNA is inserted via accidental chromoso-

116 mal integration at double stranded DNA (dsDNA) breaks through homology mediated recombi-

117 nation, non-homologous end joining repair, or a hybrid mechanism that utilizes the enzymatic

118 machinery of retrotransposons [24]. For example, inactive remnants of endogenous

119 florendoviruses (non-retroviral viruses that lack their own integrases) found across a broad diver-

120 sity of plant genomes are frequently inserted in transposable element (TE)-rich regions, specifi-

121 cally at TA dinucleotide simple repeats [27]. These repeats are thought to give rise to fragile

122 chromosome regions, making them more prone to dsDNA breaks - breaks that create potential

123 integration sites for foreign DNA.

124 Further clear evidence for the flux of genetic material from virus to host and vice versa

125 has been reported in various eukaryotes. Substantial gene exchange is particularly evident be-

126 tween the amoebozoan Acanthamoeba castellanii and its large DNA viruses [28]. 267 genes of

127 probable viral origin were identified in the genome of A. castellanii, many of which were tran-

128 scriptionally active and clearly integrated in the nuclear genome. Phylogenetic analyses also de-

129 tected a kinase gene that appears to have been transferred on multiple occasions - at least once

130 from eukaryotes to viruses and at least twice subsequently from viruses to amoebae [28]. Recent-

131 ly, experimental evidence suggested that two densovirus genes transferred to the nuclear genome

132 of pea aphids from a densovirus that infects rosy apple aphids are responsible for their ability to

133 produce winged offspring during population crowding, a phenotype with clear adaptive benefit

134 [29]. Similarly, endogenous chromosomally-transmitted bracoviruses in parasitic wasps produce

135 dsDNA circles encoding virulence genes that are injected into host caterpillars, integrate into

136 their genomes and are expressed suppressing the immune response allowing the wasp larvae to

137 survive in the lepidopteran body. These endogenous viruses appear to have been vectors for 
138 transfer of C-type lectin genes from the wasps to lepidopterans that have been domesticated by

139 the latter and allow them to resist baculovirus infection [30].

141 Transposable elements-agents of intra- and inter-genomic mobility

142 Transposable elements (TEs) have an intrinsic ability to move around within a genome,

143 which may make it easier for them to be transferred to other genomes. Numerous examples of

144 laterally transferred TEs have been reported in animals, plants, insects and fungi (e.g., [31-34]).

145 A large-scale survey of TEs in 195 diverse insect genomes identified 2,248 lateral transfers of

146 TEs, constituting 2-24\% of a given insect genome [33]. Reiss et al. [34] recently extended this

147 survey to screen 460 insect genomes for patterns of TE transfer. They concluded that DNA

148 transposons were the most frequently transferred TE class in arthropods, followed closely by

149 Long Tandem Repeats (LTRs), and that there was a clear excess of TE transfer in species hosting 150 baculoviruses.

151 With the exception of the studies mentioned above, large-sale surveys of TEs in eukary-

152 otes are generally lacking, especially in protists, making it difficult to make general conclusions

153 about the extent of inter-genomic TE mobility and its global impact on eukaryotic genome evolu-

154 tion. Regardless, studies looking at the rate of TE transfer have found that geographic proximity

155 as well as genetic distance between the host and recipient lineage impact the rate of LGT (e.g.,

156 [33]). Some of the most abundant retrotransposon families in eukaryotes were suggested to have

157 been transferred multiple times between aquatic organisms (L1) and between animals (BovB) via

158 various parasite vectors [35]. Some viral genomes contain TEs acquired from eukaryotes (e.g.

$159[23,36])$, including the widespread invasion of miniature inverted-repeat TEs found in high copy

160 numbers in viruses that use host machinery to transpose themselves [37]. Several TEs have been 
161 shown to produce extrachromosomal circular forms during transposition [38], which if

162 encapsidated in a viral particle may be more likely to undergo virus-mediated LGT than linear

163 DNA [18].

164 Under stress conditions, LTR retrotransposons are activated and generate double-stranded

165 breaks in DNA (e.g., [39]); this could potentially facilitate integration of foreign DNA at these

166 breakpoints via normal DNA repair pathways. Furthermore, imperfect excision of a TE from a

167 chromosome, as has been observed in bacteria [40], can result in large adjacent portions of the

168 genome accidentally being carried along with a TE during transposition or horizontal transfer.

169 Due to the ability of TEs to capture and transduce sequences at a high frequency, it has been

170 suggested that horizontal TE transfer could be responsible for the direct movement of genes

171 among eukaryotes as well [41]. Consistent with this hypothesis, transferred genes are often asso-

172 ciated with TE-rich regions in metazoan genomes [14]. The transfer of a wheat virulence protein

173 (ToxA) between three fungal wheat pathogens in two independent events was likely facilitated by

174 a type II DNA transposon [42]. Long-read sequencing based genome assemblies for several of

175 these fungi showed that the genomic context of the two ToxA LGTs included $14 \mathrm{kbp}$ of both cod-

176 ing and non-coding DNA, including conserved terminal inverted repeats bearing strong resem-

177 blance to features of type II DNA transposons, suggesting LGT and lateral TE transfer can occur

178 simultaneously [43]. Altogether, this strongly suggests that TEs are not only transferred laterally,

179 but that they also can facilitate the movement of other genes between species.

180

181 Endosymbiosis and gene transfer

182 A large variety of eukaryotes harbour prokaryotic and/or eukaryotic endosymbionts ex-

183 hibiting varying degrees of cellular and genomic integration. In a number of cases (e.g., mito- 
184 chondria and plastids), the endosymbionts have co-evolved with the host to the point where they

185 have become permanent, semi-autonomous organelles. In any case, the close proximity of the

186 host nucleus and endosymbionts or endosymbiont-derived organelles provides an opportunity for

187 the movement of genetic material $[43,44]$. Endosymbiont-to-host nucleus gene transfers played

188 an important role in the evolution of mitochondria and plastids, a process specifically referred to

189 as endosymbiotic gene transfer (EGT) $[45,46]$. In these instances, if the transfer of genes from

190 the endosymbiont-derived organelle to host is followed by the retargeting of the gene product to

191 the organelle, the copy of the gene in the organellar genome can be lost. In the fullness of time,

192 the result of EGT is the existence of complementary gene inventories and coordinated metabo-

193 lisms in host and endosymbiont-derived compartments [10,44]. The substrate for EGT is thought

194 to be organelle lysis; the frequency of EGT over short evolutionary timescales appears to be pos-

195 itively correlated with the number of organelles per cell (see [16,47] and references therein).

196 The extent to which bacteria other than the endosymbiotic progenitors of mitochondria

197 and plastids contributed to organelle establishment remains controversial (e.g., [10, 48-50]). In

198 the case of plastids, phylogenetic evidence suggests that LGTs from diverse bacteria - not just

199 cyanobacteria - may have facilitate organelle evolution (e.g., [51]). Beyond canonical plastids,

200 the contribution of LGT to the recently evolved 'chromatophores' in the rhizarian amoeba

201 Paulinella chromatophora is clear. Sequencing of the P. chromatophora genome revealed 228

202 nuclear genes of various bacterial affinities, only 25\% of which appear to come from the

203 cyanobacterium-derived chromatophore [52]. The suite of EGTs and LGTs, along with native

204 host nuclear genes, contribute to many essential biosynthetic pathways partially encoded by the

205 substantially reduced chromatophore genome, and encode transporters facilitating host-

206 chromatophore metabolite exchange [52,53]. The phagotrophic nature of the ancestors of $P$. 
207 chromatophora likely played an important role in sourcing these various bacterial genes, driving

208 LGT and, ultimately, chromatophore integration.

209 Various other examples of endosymbiosis-associated gene transfers have also been doc-

210 umented. Some of these transfers are directly from endosymbionts to host, while others seem to

211 be LGTs from 'third party' organisms that allow endosymbionts to lose the corresponding genes.

212 The latter is exemplified by the mealybug Planococcus citri, in which the loss of enzymes for

213 synthesizing eight amino acids in its endosymbiont Tremblaya were made possible by >20 LGTs

214 from various bacteria to the $P$. citri nuclear genome [54]; a related mealybug harbors a less

215 genomically streamlined Tremblaya endosymbiont that still encodes all the enzymes necessary

216 for the synthesis of these amino acids. This endosymbiosis actually involves a tripartite relation-

217 ship between P. citri and two bacteria - a betaproteobacterium (Tremblaya) and its

218 gammaproteobacterial endosymbiont Moranella - which exhibit complementary gene losses and

219 are themselves metabolically intertwined [54]. Peptidoglycan biosynthesis is just one pathway

220 that appears to be a mosaic of $P$. citri encoded LGTs and endosymbiont encoded genes. Using a

221 variety of techniques including Nanoscale Secondary Ion Mass Spectrometry (NanoSIMS),

222 Bublitz et al. [55] recently showed that the laterally acquired genes for peptidoglycan synthesis

223 are translated in the cytoplasm of $P$. citri and the resulting proteins are transported across multi-

224 ple membranes to the Moranella endosymbiont where peptidoglycan is assembled. These results

225 clearly show that LGT can give rise to stable, complex, mosaic biochemical pathways in

226 endosymbioses outside the bounds of mitochondria and plastids.

227 Several species of single-celled Foraminifera harbor intracellular bacteria capable of

228 denitrification - a process widespread in prokaryotes but not in eukaryotes, which allows for the

229 storage and use of nitrate in energy metabolism [56]. Recently, a novel denitrification pathway 
230 was identified in the genome of the foraminiferan Globulimina where two of the key enzymes

231 are clearly of prokaryotic origin and likely originated from the denitrifying endosymbiotic bacte-

232 ria [57]. In the ctenophore Mnemopsis leidyi, various LGTs have been suggested to be a result of

233 the association between these enigmatic invertebrates with various bacteria and protists such as

234 amoebae [58]. Numerous studies have shown a variety of LGTs from Wolbachia endosymbionts

235 into various insect and nematode hosts ([59] and references therein), ranging from smaller inser-

236 tions to genome-sized fragments. These are just a few of many examples illustrating that

237 endosymbionts contribute DNA to their hosts and that gene transfers from various sources during

238 and/or prior to the establishment of a permanent endosymbiont can influence host-endosymbiont

239 integration. The fact that EGTs have occurred in diverse lineages across the eukaryotic tree

240 shows that there are few - if any - barriers to the uptake and integration of foreign DNA into

241 eukaryote nuclear genomes.

243 "You are what you eat"

244 Many protists feed on other organisms via phagocytosis. Doolittle's 'you are what you

245 eat' hypothesis [60] posits that both prokaryotic and eukaryotic prey could act as sources of new

246 genes simply as a by-product of digestive vacuole rupture and release of nucleic acids. In this

247 respect, the overall mechanism of gene transfer from phagocytosed prey is similar to gene acqui-

248 sition from endosymbionts. Even if food vacuole or symbiont rupture is a very rare occurrence,

249 both generate free DNA that could enter the nucleus and be incorporated into the nuclear genome

250 at a low frequency. Such sequences will accumulate mutations and may be deleted. But if the

251 gene within the inserted DNA happens to be expressed, it could be fixed in the population by

252 drift or, if some advantage is conferred, positive selection. Because of their overall similarity in 
253 mechanism and outcome, it is difficult if not impossible to determine if gene transfer events orig-

254 inated from transient ancestral endosymbionts or phagocytosed food. A number of potential ex-

255 amples of the latter have nevertheless been suggested.

256 Adaptive gene transfers of bacterial origin in the bacterivorous protist Halocafeteria

257 seosinensis allow it to produce an osmoprotectant that relieves significant osmotic stress found in

258 its hypersaline habitat. This, in combination with other LGTs from bacteria, likely facilitated the

259 adaptation of $H$. seosinensis to a high salt environment [9]. In the anaerobic oxymonad

260 Monocercomonoides, a laterally acquired SUF-system from a bacterial donor (with genes veri-

261 fied to be in the nucleus by fluorescent in situ hybridization) is likely linked to the ability of

262 Monocercomonoides to ingest and digest bacterial prey [61]. This laterally acquired pathway

263 allows for iron-sulfur cluster assembly to take place in the cytosol rather than via the typical ISC

264 pathway in mitochondrion, ultimately making the last essential function of Monocercomonoides'

265 mitochondria-related-organelle redundant and dispensable [61]. Eukaryovores are relatively rare

266 compared to bacterivorous protists, which may contribute to apparent differences in the frequen-

267 cy of gene transfer between eukaryotes and between eukaryotes and prokaryotes [62]. However,

268 these differences may also reflect the difficulties associated with confidently identifying eukary-

269 ote-to-eukaryote gene transfer events (Box 3).

270

271 Parasites as LGT recipients and vectors

272 LGT has played an important role in the adaptation of many successful parasite lineages

273 to their host and interactions with other host-associated microbes. Cases of adaptive LGT from

274 prokaryotes to a variety of unicellular and multicellular parasitic eukaryotes have been well doc-

275 umented (e.g., [7,12]). For example, LGTs have been reported in diplomonad protozoa (e.g., 
277 Trichomonas and Entamoeba $[12,64]$ as well as parasitic nematodes (e.g., [14, 65]).

Several trends in the patterns of laterally transferred genes into parasitic lineages have

279 been noted. For example, there is an excess of genes received from organisms that live in the 280 same 'host' environment as the parasites (e.g., [12, 64]). Furthermore, the functions of the ac281 quired genes tend to reflect the lifestyles of the different parasite types. For example, the muco282 sal parasite Trichomonas vaginalis has acquired genes related to harvesting glycans from host 283 epithelial tissues and secretions (Box 4). A recent analysis of the anaerobic gut mucosal parasite 284 Blastocystis sp. is consistent with these observations. 2.5\% of the protein-coding genes in this 285 organism were acquired laterally from a wide range of both prokaryotes and eukaryotes, allow286 ing for successful host infection, evasion of host immune systems, modulation of other gut mi287 crobes (e.g., via production of indole and polyketides) and metabolic adaptation to the gut envi288 ronment [13]. Several of the LGTs in Blastocystis appear to have come directly from animal 289 hosts. Others appear to have been transferred multiple times between this organism and other 290 mucosal protists (e.g., Trichomonas, Giardia and Entamoeba), suggesting the potential existence 291 of a pool of adaptive genes shared between anaerobic mucosal parasites. Numerous other strong 292 examples of LGT facilitating parasite adaptation have been reported across the eukaryotic tree.

293 For example, Dean et al. [66] used a combination of ancestral gene reconstruction and functional 294 assays to show that the acquisition of a single nucleotide transporter from bacteria to the com295 mon ancestor of Microsporidia species allowed them to become energy parasites that steal host 296 ATP, GTP and NAD+ and subsequently lose the capacity to synthesize nucleotides on their own. 297 In phytoparasitic nematodes, functionally important genes encoding enzymes involved in 298 plant cell-wall degradation and related metabolism were identified as LGTs from bacteria that 
299 are plant pathogens of the same hosts, as well as from a variety of eukaryotes (including the plant

300 pathogen Phytophthora infestans) [14]. Interestingly, LGTs in these nematodes are often found

301 in TE regions of the genome, and the bacterial homologs are found on plasmids, suggesting a

302 mechanistic role for mobile genetic elements in LGT. Another example of host-parasite mediated

303 LGT is the acquisition of mammalian TE families by the blood-sucking insect Rhodnius prolixus

304 and the pond snail Lymnaea stagnalis. These TEs had extremely high sequence identities to TEs

305 in the genomes of host mammals of the insect and trematode parasites for which the snail acts as

306 an intermediate host [67], implicating host-parasite interactions in horizontal transmission of the

307 TEs. Based on the foregoing examples, it seems likely that parasites that infect a wide range of

308 hosts or species that exist in general symbiotic associations could be particularly potent vectors

309 for LGT.

310

\section{Conjugation and natural transformation in eukaryotes}

312 A wide variety of eukaryotes have been transformed in the lab. While artificial in nature,

313 such experiments indicate that there is no fundamental obstruction to LGT when barriers to

314 transfer are intentionally removed. Established eukaryotic transformation systems are frequently

315 exploited for commercial applications such as the production of genetically modified crops. For

316 example, Agrobacterium tumefaciens, which uses a type IV secretion system to inject a tumor

317 inducing (Ti) plasmid carrying specific genes into plant cells, has been used to modify plant spe-

318 cies to confer pest resistance [68]. Once in the host cell, the Ti plasmid utilizes the plants ma-

319 chinery to enter the nucleus and integrate into the genome, using eukaryotic promoters to express

320 its genes. Importantly, there is evidence that this transformation occurs naturally in plants as

321 well. Genes from Agrobacterium have been found to reside and be expressed in the germ line of 
322 various plant species that have not been artificially transformed (e.g., $[69,70])$, suggesting that 323 these gene transfers are maintained and functional.

324 Recently a $100 \%$ efficient transformation system was developed in the red alga

325 Porphyridium purpueum where the transformation vectors are maintained episomally as

326 extrachromosomal high-copy number plasmids [71]. This shows that different bacterial origins

327 of replication can function in a eukaryote system and result in stable, autonomous replication

328 within the nucleus. Naturally occurring extrachromosomal plasmids of unknown origin have

329 been found in some red algae; these plasmids contain both bacterial and viral ORFs (e.g., [72]).

330 The apparent genetic compatibility between plasmid replication systems of bacteria and compo-

331 nents of DNA replication machinery in the red algal nucleus [73] raises intriguing questions

332 about algal genome evolution. The extended presence and replication of a nuclear episomal ele-

333 ment could provide opportunities for gene transfer to the nuclear genome and may have facilitat-

334 ed the contribution of bacterial genes to red algae (estimated to be between $1 \%$ and $9.3 \%$ of the

335 total gene content, depending on the lineage $[73,74])$.

336 Interestingly, a conjugation-based transformation system capable of delivering episomal

337 vectors from E. coli has been developed for the diatoms Phaeodactylum tricornutum and

338 Thalassiosira pseudonana [75]. Although direct conjugation has not been observed in nature,

339 this system suggests that bacterial conjugation may be one mechanism that could explain the

340 high percentage of putative bacterial-derived genes (5-10\% of genes) in diatom genomes [76].

341 The transfer of plasmids via bacterial conjugation has been observed in yeast [77], as has the

342 natural transformation of exogenous DNA under non-artificial conditions [78].

\section{Difficulties in detecting LGT in eukaryotes}


Eukaryotes typically have relatively large and complex genomes compared to prokary-

346 otes, making genome sequencing, assembly and analysis difficult. The paucity of high-quality

347 genome data from diverse eukaryotes, especially from closely related species and multiple

348 strains of the same species, is a present barrier to the study of LGT [79]. In regions of the eukar-

349 yotic tree in which there is denser genomic sampling of lineages, the extent of adaptive LGT is

350 becoming clearer. For example, Ropars et al. [80] analyzed 10 high-quality genomes from

351 Penicillum in the context of genome data from 416 strains of 65 fungal species. The authors con-

352 fidently identified 104 LGTs between Penicillium species, including some large recently trans-

353 ferred genomic regions spanning more than $10 \mathrm{~kb}$, some of which show signatures of TE mediat-

354 ed insertion [80]. Gene expression analysis and fitness experiments confirmed that at least some

355 of the LGTs encode functional genes that provide metabolic adaptations to a cheese environment

356 (e.g. lactose metabolism) or antifungal proteins used to out-compete other fungal species [80].

357 Until a similar density of high-quality genomic sampling is available for other branches of the

358 eukaryote tree- particularly for microbial eukaryote lineages - it is impossible to know whether

359 the dynamic nature of fungal genomes is the exception or the rule.

360 Distinguishing true LGTs from contamination (see 81) can be difficult and mitigated by

361 careful inspection of candidate genes (Box 3). Many (but by no means all) published studies

362 have used appropriately stringent phylogenetic criteria for reporting LGT. Initial bioinformatic

363 steps generally lean towards the exclusion of bacterial/symbiont sequences from assemblies,

364 which means that the impact of LGT to a given genome can be underestimated. Additional diffi-

365 culties arise when trying to determine the scale of how far back in evolutionary time one has to

366 go to consider a gene as having been vertically or laterally transferred. More ancient LGTs are

367 harder to detect as the sequence similarity of a transferred gene to the gene of the donor lineage 
368 decays over time. At the same time, confidently detecting eukaryote-eukaryote LGT is generally

369 more difficult than prokaryote-eukaryote LGT due to the more recent common ancestry shared

370 between donor and recipient, resulting in less obvious phylogenetic incongruence. A 'patchy'

371 gene distribution across the eukaryote tree is often interpreted as evidence of LGT between eu-

372 karyotic lineages. However, secondary loss of ancestral genes is well known to have occurred in

373 parallel in many eukaryotic lineages (e.g. genes in mitochondrial genomes, reviewed in [10]) and

374 is sometimes an equally plausible alternative explanation for the gene distribution pattern.

375 Therefore, strongly supported single gene trees that contradict the expected species tree relation-

376 ships are needed to support suspected LGT cases. This standard is sometimes challenging to

377 achieve due to the limited phylogenetic signal within many single gene alignments. Identifying

378 gene transfers in eukaryotes also requires a sufficient level of taxonomic sampling of the given

379 gene of interest, which is often lacking. Strategic sequencing of diverse eukaryote lineages will

380 make LGT detection easier in the future.

382 LGT and pan-genomes in eukaryotes

383 In prokaryotes, within-species variation in gene content is such that the core genome (i.e.,

384 gene families found across all or most strains) represents 3-84\% of the total gene families found

385 in all sequenced strains of a given species (reviewed by [3]). The accessory genome includes

386 genes found in a single or subset of strains (Figure 2). This pan-genome pattern is due to the

387 combined effects of LGT and differential gene loss.

388 The extent to which a pan-genome-like pattern is observed in various eukaryote lineages

389 - and, in particular, protists - is less clear, as inferences are limited by the paucity of genomes

390 that cover the breadth of diversity for a given species outside of animals, plants and fungi [79]. 
391 Another problem is the difficulty in obtaining accurate and comprehensive gene predictions in

392 eukaryotes, as gene prediction in much more difficult than in prokaryotes because of the pres-

393 ence of spliceosomal introns and alternative splicing. As a result, potential pan-genome patterns

394 must be cross-checked to be certain they are not simply artefacts of failure to predict existing

395 genes. Nevertheless, across various model fungal species where a dozen or more genomes have

396 been sequenced, pan-genomes have been observed with core genomes ranging from $80-90 \%$ of

397 the species coding capacity [e.g., 82 and references therein]. Some of the accessory genes are

398 inferred to be a result of LGT; others appear to be a consequence of gene duplication,

399 pseudogenization and outright gene loss. In plants, the accessory genome component of the spe-

400 cies pan-genome seems to be more extensive (up to $\sim 47 \%$ of the pan-genome). For example,

401 across >3,000 genomes of Oryza sativa L., $46.5 \%$ of the pan-genome was found to be accessory

402 genes, most of which are related to immune and defense responses [83].

403 In the world of protists and algae, fascinating insight into the eukaryotic pan-genome has

404 come from investigation of the coccolithophorid Emiliania huxleyi [84]. In analyzing the ge-

405 nomes of 14 highly-similar strains of E. huxleyi (>98\% 18S ribosomal DNA (rDNA) identity),

406 Read et al. [84] found a core genome (experimentally confirmed via microarrays) comprised of

407 only $69.5 \%$ of the total gene set. While Read et al. were unable to confidently assign LGTs from

408 bacteria or viruses as the main source of the accessory genes, they did find that E. huxleyi ge-

409 nomes contain a lot of repetitive elements, which may foster accessory gene formation and serve

410 as preferential target sites for LGT.

411 As with prokaryotes, there are serious problems in defining the 'pan-genome' because it

412 is confounded with how species are defined and delimited. For example, Worden et al. [85]

413 found significant gene content differences between two isolates of Micromonas that were origi- 
414 nally considered the same species on the basis of morphology and 18S rDNA similarity. The fact

415 that these two isolates were found to share only $90 \%$ of their genes was interpreted to mean that

416 they were different species. Yet, an alternative interpretation is that these two isolates are mem-

417 bers of the same pan-genome-containing species. In the absence of evidence for sexual recombi-

418 nation between individuals of a putative species - information that is lacking for the majority of

419 protists - defining a species is difficult. Without a clear species definition for most microbial 420 eukaryotes, it is difficult to distinguish between these two scenarios.

\section{Concluding Remarks}

423 The past few years have seen a rapid increase in the sequencing of complete genomes

424 from diverse eukaryotes and, concordantly, abundant evidence for LGT into eukaryotic genomes.

425 Many questions nevertheless remain unanswered (see Outstanding Questions). Indeed, for most

426 documented cases that are not obviously EGTs, the means by which the transferred DNA was

427 introduced into cell and incorporated into the chromosomes remain unclear. Many of the mecha-

428 nisms that could have been involved, and their relative importance, are 'known unknowns'. For

429 example, endosymbiont- or 'you-are-what-you-eat' mediated transfers are probably much more

430 common in eukaryotes that commonly harbor endosymbionts and phagocytose prey, respective-

431 ly. On the other hand, virus-mediated transfer will depend on the number and types of viruses

432 that infect particular lineages and the degree to which they can integrate into host genomes and

433 mobilize host DNA. To address these questions the following data are needed. First, a greater

434 density of sampling of eukaryotic genomes from each of the major eukaryotic groups should

435 allow us to 'catch' more very recent transfer events that may still bear the hallmarks of their ori-

436 gins (e.g., flanking TEs, viral genes, breakpoints of recombination events etc.). This should also 
437 allow us to assess the extent to which pan-genomes are a common feature of diverse eukaryote

438 species, assuming we can get reliable information about how such species are delimited (i.e.,

439 evidence for recombination within species). Our understanding of eukaryote LGT will also be

440 greatly enhanced by the development of new model systems and long-term laboratory evolution

441 experiments that allow the process of LGT to be studied in real time. Ideally, organisms from

442 different branches of the eukaryotic tree should be developed as model systems and the various

443 insights they yield considered in isolation and together. Long-term progress in our understanding

444 of the frequency and significance of LGT in eukaryotes will rely on the synthesis of information

445 gleaned from both experimental and bioinformatic approaches.

\section{$447 \quad$ References}

4481 Soucy, S. et al. (2015) Horizontal gene transfer: building the web of life. Nat. Rev. Genet. 16, $449 \quad 472-482$

4512 Land, M. et al. (2015) Insights from 20 years of bacterial genome sequencing. Funct. Integr. 452 Genomics 15, 141-161

4543 McInerney, J.O. et al. (2017) Why prokaryotes have pangenomes. Nat. Microbiol. 2, 17040

4 Vos, M. and Eyre-Walker, A. (2017) Are pangenomes adaptive or not? Nat. Microbiol. 2, 1576-1576

460 BMC Biol. 14, 101

4626 Leger, M.M. et al. (2018) Demystifying eukaryote lateral gene transfer. BioEssays 40, $463 \quad 1700242$

4657 Lukeš, J. and Husník, F. (2018) Microsporidia: a single horizontal gene transfer drives a great 466 leap forward. Curr. Biol. 28, R712-R715 
an extremophilic eukaryote. Science 339, 1207-1210

9 Harding, T. et al. (2017) Adaptations to high salt in a halophilic protist: differential expression and gene acquisitions through duplications and gene transfers. Front. Microbiol. 8, 944

10 Roger, A.J. et al. (2017) The origin and diversification of mitochondria. Curr. Biol. 27,

11 Stairs, C.W. et al. (2018) Microbial eukaryotes have adapted to hypoxia by horizontal acquisitions of a gene involved in rhodoquinone biosynthesis. eLife 7, e34292

12 Hirt, R.P. et al. (2015) Lateral gene transfers and the origins of the eukaryote proteome: a view from microbial parasites. Curr. Opin. Microbiol. 23, 155-162

13 Eme, L. et al. (2017) Lateral gene transfer in the adaptation of the anaerobic parasite Blastocystis to the gut. Curr. Biol. 27, 807-820

14 Paganini, J. et al. (2012) Contribution of lateral gene transfers to the genome composition and parasitic ability of root-knot nematodes. PLoS One 7, e50875

$15 \mathrm{Xu}$, F. et al. (2016) On the reversibility of parasitism: adaptation to a free-living lifestyle via gene acquisitions in the diplomonad Trepomonas sp. PC1. BMC Biol. 14, 62

16 Bock, R. (2017) Witnessing genome evolution: experimental reconstruction of endosymbiotic and horizontal gene transfer. Annu. Rev. Genet. 51, 1-22

17 Strese, A. et al. (2014) A recently transferred cluster of bacterial genes in Trichomonas vaginalis - lateral gene transfer and the fate of acquired genes. BMC. Evol. Biol. 14, 119

18 Gilbert, C. and Cordaux, R. (2017) Viruses as vectors of horizontal transfer of genetic materi-

19 Longdon, B. et al. (2014) The evolution and genetics of virus host shifts. PLoS Pathog. 10,

20 Catoni, M. et al. (2018) Virus-mediated export of chromosomal DNA in plants. Nat.

505 Commun. 9, 5308 
51022 Moreira, D. and Brochier-Armanet, C. (2008) Giant viruses, giant chimeras: the multiple evo511 lutionary histories of Mimivirus genes. BMC Evol. Biol. 8, 12

51323 Gilbert, C. et al. (2016) Continuous influx of genetic material from host to virus populations. $514 \quad$ PLoS Gene. 12, e1005838 impact on host biology. Nat. Rev. Genet. 13, 283-296

25 Marchi, E. et al. (2014) Unfixed endogenous retroviral insertions in the human population. J.

520 Virol. 88, 9529-9537

26 Filée, J. (2014) Multiple occurrences of giant virus core genes acquired by eukaryotic genomes: The visible part of the iceberg? Virology 466, 53-59

27 Geering, A.D. et al. (2014) Endogenous florendoviruses are major components of plant genomes and hallmarks of virus evolution. Nat. Commun. 5, 5269

29 Parker, B.J. and Brisson, J.A. (2019) A laterally transferred viral gene modifies aphid wing

532

533

30 Gasmi, L. et al. (2015) Recurrent domenstication by Lepidoptera of genes from their parasites medicated by bracoviruses. PLoS Genet. 11:e1005470

31 Hill, T. and Betancourt, A.J. (2018) Extensive exchange of transposable elements in the Drosophila pseudoobscura group. Mobile DNA 9, 20 
35 Ivancevic, A.M. et al. (2018) Horizontal transfer of BovB and L1 retrotransposons in eukaryotes. Genome Biol. 19, 85

36 Gilbert, C. et al. (2014) Population genomics supports baculoviruses as vectors of horizontal transfer of insect transposons. Nat. Commun. 5, 3348

37 Zhang, H. et al. (2018) Unexpected invasion of miniature inverted-repeat transposable elements in viral genomes. Mobile DNA 9, 19

38 O'Brochta, D.A. et al. (2009) Transpositionally active episomal hAT elements. BMC Mol. Biol. 10, 1-15

39 Hedges, D.J. and Deininger, P.L. (2007) Inviting instability: Transposable elements, doublestrand breaks, and the maintenance of genome integrity. Mutat. Res. 616, 46-59

40 Frost, L.S. et al. (2005) Mobile genetic elements: the agents of open source evolution. Nat. Rev. Microbiol. 3, 722-732

41 Schaack, S. et al. (2010) Promiscuous DNA: horizontal transfer of transposable elements and why it matters for eukaryotic evolution. Trends Ecol. Evol. 25, 537-546

42 McDonald, M.C. et al. (2019) Transposon-mediated horizontal transfer of the host-specific

virulence protein ToxA between three fungal wheat pathogens. mBio 10, e01515-19

43 Sieber, K.B. et al. (2017) Lateral gene transfer between prokaryotes and eukaryotes. Exp. Cell Res. 358, 421-426

44 Husnik, F. and McCutcheon, J.P. (2017) Functional horizontal gene transfer from bacteria to eukaryotes. Nat. Rev. Microbiol. 16, 67-79

45 Martin, W. et al. (1993) Evidence for a chimeric nature of nuclear genomes: eubacterial origin of eukaryotic glyceraldehyde-3-phosphate dehydrogenase genes. Proc. Natl. Acad. Sci. U.S.A. 90, 8692-8696

46 Timmis, J.N. et al. (2004) Endosymbiotic gene transfer: organelle genomes forge eukaryotic 
$48 \mathrm{Ku}, \mathrm{C}$. et al. (2015) Endosymbiotic gene transfer from prokaryotic pangenomes: Inherited chimerism in eukaryotes. Proc. Natl. Acad. Sci. U.S.A. 112, 10139-10146

49 Archibald, J.M. (2015) Genomic perspectives on the birth and spread of plastids. Proc. Natl. Acad. Sci. U.S.A. 112, 10147-10153

50 Pittis, A.A. and Gabaldón, T. (2016) Late acquisition of mitochondria by a host with

51 Qiu, H. et al. (2013) Assessing the bacterial contribution to the plastid proteome. Trends Plant Sci. 18, 680-687

52 Nowack, E.C. et al. (2016) Gene transfers from diverse bacteria compensate for reductive 602 genome evolution in the chromatophore of Paulinella chromatophora. Proc. Natl. Acad. Sci. U.S.A. $113,12214-12219$

53 Singer, A. et al. (2017) Massive protein import into the early-evolutionary-stage photosynthetic organelle of the amoeba Paulinella chromatophora. Curr. Biol. 27, 2763-2773

54 Husnik, F. et al. (2013) Horizontal gene transfer from diverse bacteria to an insect genome enables a tripartite nested mealybug symbiosis. Cell 153, 1567-1578

56 Kamp, A. et al. (2015) Nitrate storage and dissimilatory nitrate reduction by eukaryotic mi55 Bublitz, D.C. et al. (2019) Peptidoglycan production by an insect-bacterial mosaic. Cell 179, 703-712

57 Woehle, C. et al. (2018) A novel eukaryotic denitrification pathway in foraminifera. Curr. crobes. Front. Microbiol. 6, 1492

58 Hernandez, A.M. and Ryan, J.F. (2018) Horizontally transferred genes in the ctenophore Mnemiopsis leidyi. PeerJ 6, e5067

59 Doudoumis, V. et al. (2012) Tsetse-Wolbachia symbiosis: comes of age and has great potential for pest and disease control. J. Invertebr. Pathol. 112, S94-S103

rial genes in eukaryotic nuclear genomes. Trends Genet. 14, 307-311 
61 Karnkowska, A. et al. (2016) A eukaryote without a mitochondrial organelle. Curr. Biol. 26, $630 \quad 1274-1284$

62 Keeling, P.J. and Palmer, J.D. (2008) Horizontal gene transfer in eukaryotic evolution. Nat.

63 Andersson, J.O. et al. (2007) A genomic survey of the fish parasite Spironucleus salmonicida indicates genomic plasticity among diplomonads and significant lateral gene transfer in eukaryote genome evolution. BMC Genomics 8, 51

64 Alsmark, C. et al. (2013) Patterns of prokaryotic lateral gene transfers affecting parasitic microbial eukaryotes. Genome Biol. 14, R19

65 Mayer, W.E. et al. (2011) Horizontal gene transfer of microbial cellulases into nematode genomes is associated with functional assimilation and gene turnover. BMC Evol. Biol. 11, 13 Microsporidia intracellular parasites. Nat. Commun. 9, 1709

69 Matveeva, T.V. et al. (2012) Horizontal gene transfer from genus Agrobacterium to the plant Linaria in nature. Mol. Plant-Microbe Interact. 25, 1542-1551

70 Kyndt, T. et al. (2015) The genome of cultivated sweet potato contains Agrobacterium T658 DNAs with expressed genes: An example of a naturally transgenic food crop. Proc. Natl. Acad. 659 Sci. U.S.A. $112,5844-5849$

$71 \mathrm{Li}, \mathrm{Z}$. and Bock, R. (2018) Replication of bacterial plasmids in the nucleus of the red alga red algal genomes. Sci. Rep. 6, 23744 
67074 Rossoni, A.W. et al. (2019) The genomes of polyextremophilic cyanidiales contain $1 \%$ hori-

671 zontally transferred genes with diverse adaptive functions. eLife 8, e45017

67375 Karas, B.J. et al. (2015) Designer diatom episomes delivered by bacterial conjugation. Nat.

674 Commun. 6, 6925

675

76 Bowler, C. et al. (2008) The Phaeodactylum genome reveals the evolutionary history of dia-

677 tom genomes. Nature 456, 239-244

78 Nevoigt, E. et al. (2000) Cells of the yeast Saccharomyces cerevisiae are transformable by 683 DNA under non-artificial conditions. Yeast 16, 1107-1110

79 Sibbald, S.J. and Archibald, J.M. (2017) More protist genomes needed. Nat. Ecol. Evol. 1, 145

80 Ropars, J. et al. (2015) Adaptive horizontal gene transfers between multiple cheese-associated fungi. Curr. Biol. 25, 2562-2569

81 Richards, T.A. and Monier, A. (2016) A tale of two tardigrades. Proc. Natl. Acad. Sci. U.S.A. $113,4892-4894$ 82 McCarthy, C.G. and Fitzpatrick, D.A. (2019) Pan-genome analyses of model fungal species. Microb. Genomics DOI: 10.1099/mgen.0.000243

84 Read, B.A. et al. (2013) Pan genome of the phytoplankton Emiliania underpins its global dis-

tribution. Nature 499, 209-213

85 Worden, A.Z. et al. (2009) Green evolution and dynamic adaptations revealed by genomes of the marine picoeukaryotes Micromonas. Science 324, 268-272 2283 
87 Savory, F. et al. (2018) Ancestral function and diversification of a horizontally acquired oomycete carboxylic acid transporter. Mol. Biol. Evol. 35, 1887-1900

88 Milner, D.S. et al. (2019) Environment-dependent fitness gains can be driven by horizontal gene transfer of transporter-encoding genes. Proc. Natl. Acad. Sci. U.S.A. 116, 5613-5622

89 Kominek, J. et al. (2019) Eukaryotic acquisition of a bacterial operon. Cell 176, 1356-1366

90 Dunning, L.T. et al. (2019) Lateral transfers of large DNA fragments spread functional genes among grasses. Proc. Natl. Acad. Sci. U.S.A. 116, 4416-4425

$91 \mathrm{McVey}, \mathrm{M}$. and Lee, S. (2008) MMEJ repair of double-strand breaks (director's cut): deleted sequences and alternative endings. Trends Genet. 24, 529-538

92 Huang, J. (2013) Horizontal gene transfer in eukaryotes: The weak-link model. BioEssays 35, $868-875$

93 Matsuo, M. et al. (2018) Characterization of spliced leader trans-splicing in a photosynthetic rhizarian amoeba, Paulinella micropora, and its possible role in functional gene transfer. PLoS One 13, e0200961

94 Doolittle, W. et al. (2003) How big is the iceberg of which organellar genes in nuclear genomes are but the tip? Philos. Trans. R. Soc. London, Ser. B 358, 39-58

95 Vembar, S. et al. (2016) Complete telomere-to-telomere de novo assembly of the Plasmodium falciparum genome through long-read $(>11 \mathrm{~kb})$, single molecule, real-time sequencing. DNA Res. 23, 339-351

96 Funkhouser-Jones, L.J. et al. (2015) Wolbachia co-infection in a hybrid zone: discovery of horizontal gene transfers from two Wolbachia supergroups into an animal genome. PeerJ 3 , e1479

97 Brelsfoard, C. et al. (2014) Presence of extensive Wolbachia symbiont insertions discovered in the genome of its host Glossina morsitans morsitans. PLoS Negl. Trop. Dis. 8, e2728

98 Pinheiro, J. et al. (2018) The protozoan Trichomonas vaginalis targets bacteria with laterally acquired NlpC/P60 peptidoglycan hydrolases. mBio 9, e01784-18

99 Pereira-Neves, A. and Benchimol, M. (2007) Phagocytosis by Trichomonas vaginalis: new insights. Biol. Cell 99, 87-101

100 Liu, L. et al. (2012) The human microbiome: A hot spot of microbial horizontal gene trans- 
752 fer. Genomics 100, 265-270

753

754101 Parfrey, L. et al. (2011) Microbial eukaryotes in the human microbiome: ecology, evolution, 755 and future directions. Front. Microbiol. 2, 153

\section{Acknowledgements}

763 We thank the reviewers for a number of constructive suggestions and apologize to authors whose

764 work could not be cited due to length constraints. SS was supported by a CGS-D scholarship

765 from the Natural Sciences and Engineering Research Council of Canada. The work was also

766 supported by a Foundation Grant, FRN-142349, from the Canadian Institutes of Health Research

767 awarded to AJR, a Symbiosis Grant from the Moore Foundation awarded to JMA and LE is sup-

768 ported by funding from the European Research Council (ERC) under the European Union's

769 Horizon 2020 research and innovation programme (grant agreement No 803151).

771 Glossary

\section{Transduction}

773 Virus-mediated transfer of genetic material. In bacteria, generalized transduction involves the

774 integration and transfer of random pieces of bacterial DNA into the viral genome, while in spe-

775 cialized transduction only defined regions of the donor genome can be packaged into the virus

776 and transferred. 
779 Uptake of exogenous DNA by a cell. This can occur naturally or in an experimental context.

780 Both prokaryotic and eukaryotic cells are capable of being transformed.

\section{Conjugation}

783 Transfer of genetic material mediated by cell-cell contact; single-stranded DNA moves through a 784 thin connection between the donor and recipient cells. Although best known to occur between 785 bacteria, artificial conjugation between bacteria and eukaryotes such as diatoms has been carried 786 out.

788 Pan-genome

789 The term refers to the entire set of genes in the genomes of members of a given species. The

790 word was invented to underscore the fact that individuals or strains of a given species can vary in

791 their gene content. In bacterial and archaeal species genome content variability can be immense

792 such that the set of genes found in all members of a given species (the 'core') can represent a

793 small fraction of the total pan-genome. This variability in gene content has a number of causes

794 including gene duplications, gene losses, de novo gene formation and the acquisition of new 795 genes by lateral gene transfer (LGT).

\section{Protists}

798 Protists are any eukaryotic organisms that are not animals, plants or fungi. Protists are an ances-

799 tral 'grade' of eukaryotic organization and encompass the bulk of eukaryotic diversity. Although 
800 most protist species are unicellular microbes, charismatic multicellular forms are well known

801 (e.g., macroalgae like giant kelp).

802

\section{Endosymbiont}

804 An organism that thrives within another organism, typically without killing its host. Endosym-

805 biosis of bacteria, archaea and microbial eukaryotes (e.g., algae) is relatively common in free-

806 living protists, but more rare in parasites. Organelles such as mitochondria and chloroplasts

807 evolved from ancient bacterial endosymbionts. Readers should keep in mind that (en-

808 do)symbiosis encompasses a continuum from mutualism to parasitism, and determining where a 809 given symbiotic system lies along this continuum can be very difficult.

810

\section{Endosymbiotic gene transfer (EGT)}

812 The transfer of genetic material from endosymbionts and their organellar derivatives to the host

813 cell nuclear genome. This process involves endosymbiont / organelle lysis, migration of released

814 DNA into the nucleus, and integration of random pieces of foreign DNA into nuclear chromo-

815 somes by DNA repair enzymes.

816 


\section{Box 1: Robust examples of LGT in eukaryotes}

This review focuses on a select few of the numerous reports of LGT in eukaryotes. A

819 recent example of adaptive LGT concerns the acquisition of the gene rquA, which is involved in

820 rhodoquinone (RQ) biosynthesis, in various anaerobic protistan lineages [11]. The RQ produced

821 by RquA allows for fumarate to be used as an alternative terminal electron acceptor during mito-

822 chondrial ATP production under anaerobic conditions, allowing these protists to adapt to low

823 oxygen conditions. Phylogenetic analyses suggest that $r q u A$ was transferred on at least two sepa-

824 rate occasions from bacteria to eukaryotes, followed by multiple eukaryote-to-eukaryote trans-

825 fers.

826 Single gene-encoded transporters may be especially amenable to LGT as they could pro-

827 vide a direct selective advantage to the recipient by allowing for the uptake and utilization of a

828 particular metabolite. Numerous examples of LGTs involving transporter genes have been re-

829 ported (e.g., [68,86-88 and references therein]). Milner et al. [88] identified seven cases of trans-

830 porter-gene LGT between fungal phyla and demonstrated that they conferred a competitive ad-

831 vantage in certain environments. They proposed a "transporter gene acquisition ratchet" where

832 the range of transporters encoded in a given genome are continually being altered by various

833 mechanisms, including LGT.

834 Another notable report of LGT is the acquisition of an entire operon involved in

835 siderophore biosynthesis from Enterobacteriaceae into a group of closely related yeasts [89]. The

836 co-linearly arranged genes were found to be expressed and functional (via transcriptomics, meta-

837 bolic assays, and mass spectrometry), allowing for the sequestering and uptake of iron from the

838 environment - a likely beneficial feature as the yeast reside in the iron-limited insect gut envi-

839 ronment co-inhabited with numerous Enterobacteriaceae. After the initial LGT, the operon struc- 
840 ture appears to have undergone a 'eukaryotification' process that included the addition of

841 polyadenylation sites, various structural rearrangements, addition of some eukaryotic genes, and

842 loss of some genes encoded by the operon in closely related yeast lineages. In principle, acquir-

843 ing an entire operon in a single LGT would allow for complex multi-gene pathways to spread

844 rapidly and effectively from donor to recipient.

845 Analyses of chromosome-level genomes for the grass Alloteropsis semialata also re-

846 vealed recent acquisitions of chunks of foreign DNA encoding multiple genes, including genes

847 related to disease resistance, abiotic stress tolerance and C4 photosynthesis [90]. Phylogenetic

848 analyses including genomes from >140 other grass species led to the discovery of 59 unambigu-

849 ous, recent LGTs into A. semialata from at least nine distantly related donor grasses. These genes

850 were found to be mostly expressed and clustered in 23 transferred fragments up to $170 \mathrm{kbp}$ long,

851 indicating that large stretches of DNA containing multiple functional genes as well as non-

852 coding regions were transferred in a single event. In some cases, TEs were also encoded on the 853 transferred region.

855 Box 2: Factors influencing the frequency of lateral gene transfer

856 The frequency of gene transfer into a given species may hinge on a number of genomic

857 and environmental factors. For example, non-homologous end joining repair could be a major 858 mechanism of incorporation of foreign DNA [44] and thus impact the frequency of LGT in a 859 given genome. It is generally thought that successful LGTs can occur more frequently between 860 closely related organisms due to similarities between their genomes (codon usage, cis-acting 861 regulatory elements, etc.); high levels of sequence identity also foster homologous recombina862 tion, which can lead to the transfer of alleles from one species to another [1]. Genetic exchange, 
863 however, is not completely limited to very similar sequences. As long as there are conserved

864 flanking regions, divergent alleles can recombine by homologous recombination [1] and the

865 presence of small short repeats may allow for micro-homology mediated recombination as well

866 [91].

867 Dynamic and repetitive regions of the genome that are more prone to duplication are like-

868 ly to have increased rates of gene integration. LGTs have been observed in regions enriched in

869 DNA transposons and retrotransposons and in gene-poor regions such as telomeres or in/near

870 TEs (discussed in [43]). Additionally, selfish genetic elements (e.g., self-splicing introns, inteins

871 etc.) are common in multicellular eukaryotes and could promote LGT and genome rearrange-

872 ments [1]. 'Fragile' genomic regions that are prone to double stranded breaks or environmentally

873 induced DNA damage and repair could also create opportunities for foreign gene integration.

874 LGTs may occur when viruses or parasites manipulate DNA damage/repair pathways. Certain

875 life/developmental stages may be more likely entry points for foreign genes into the nuclei of 876 both unicellular eukaryotes (particularly those who are not phagotrophic), and in particular, the

877 germline of multicellular eukaryotes (see the 'weak-link' model [92]).

878 In the testate amoeba Paulinella micropora, Matsuo et al. [93] identified a common $~ 30$

879 bp sequence added post-transcriptionally by spliced leader (SL) trans-splicing to a diverse set of

880 mRNAs, including those derived by LGT and EGT. Based on these observations, the authors

881 suggested that SL trans-splicing could mitigate the potential detrimental effects of LGT in eu-

882 karyotes by ensuring translation of native nuclear genes disrupted by the insertion of foreign

883 genes in their 5'UTRs. This could in principle apply not only to Paulinella species but to other

884 trans-splicing organisms including euglenids, dinoflagellates and some metazoans. 


\section{Box 3: Detecting LGT with confidence}

Genomic features that differ between eukaryotic and prokaryotic genes can be used to differentiate between bona fide LGT and prokaryotic contaminants. One useful distinction is the

889 presence of introns in eukaryotic genes and lack thereof in prokaryotes; if a candidate LGT of

890 bacterial origin contains an intron it most likely resides within a eukaryote genome. Other char-

891 acteristics such as nucleotide composition, amino acid usage and the presence of a poly-A tail on

892 transcripts can help identify genuine LGTs. Further support can be found by examining the ge-

893 nomes of closely related organisms; unless the transfer is very recent, the suspected LGT will

894 likely be found in multiple genomes. For example, most of the LGTs identified in the gut para-

895 site Blastocystis sp. subtype 1 were also found in genomes of seven of the other subtypes [13].

896 Phylogenetic analysis is essential for differentiating candidate LGTs from gene duplica-

897 tions and differential loss. A patchy gene distribution, combined with a phylogenetic relationship

898 that contradicts the species relationship, is suggestive of an LGT. If it is assumed that all patchily

899 distributed eukaryotic genes are due to differential gene loss rather than lateral acquisitions, then

900 there becomes a genome of Eden problem [94] - the unlikely scenario that the last eukaryotic

901 common ancestor (LECA) had almost every gene seen in modern eukaryotes and an enormous

902 range of metabolic capabilities. Phylogenetic reconstruction of LGT is not without its limitations,

903 with certain transfers being more difficult to detect and interpret (such as sequential transfers,

904 which results in more complex gene distributions [62]). The lack of resolution associated with

905 single gene trees can make it tough to infer the validity and origin of candidate LGTs. Phyloge-

906 netic artifacts, leading to falsely resolved relationships that may mislead interpretations of a

907 gene's evolutionary history, are also of concern. 
909 tigations of genomic context. A candidate LGT contained within a larger genomic scaffold clear-

910 ly of host origin is likely to be real (e.g., viral LGTs in Acanthamoeba that are surrounded by

911 amoebal host genes [28]). This can be difficult to assess if a reference genome is poorly assem-

912 bled and highly fragmented. Fortunately, long read sequencing technologies make it possible to

913 produce more highly contiguous genome assemblies, allowing for greater insight into genomic

914 context, particularly in repetitive regions and near telomeric repeats that typically do not assem-

915 ble well (e.g., [95]). Classical molecular biology techniques can also be used to verify genomic

916 integration of candidate LGTs into host chromosomes (e.g., fluorescence in situ hybridization

917 (FISH) has been used to confirm insertions of Wolbachia DNA on multiple chromosomes in the

918 grasshopper Chorthippus paralleulus and Tsetse flies (Glossina spp.) [96, 97].

920 Box 4: Trichomonas and gene transfer in the microbiome

921 Trichomonas vaginalis is a sexually transmitted parasite that colonizes an environment

922 occupied by other parasites and bacteria. 149 LGTs of both prokaryotic and eukaryotic origin

923 were identified in the T. vaginalis genome, including genes likely facilitating pathogenicity and

924 to take advantage of the rich metabolic opportunities in its environment (e.g., glycan degrada-

925 tion) [64]. Further study on LGTs in T. vaginalis identified and confirmed the functionality of

926 NIPC/P60 peptidoglycan hydrolases that were acquired laterally from bacterial co-members of

927 the microbiota [98]. Pinheiro et al. [98] showed that the bacterial NIPC/P60 hydrolases in the

928 genome of T. vaginalis were capable of degrading bacterial cell envelopes and that their expres-

929 sion was significantly upregulated in the presence of bacteria. The transfer of NIPC/P60 hydro-

930 lases in $T$. vaginalis appears to be critical in controlling bacterial abundance in its environment 
931 and the establishment of a successful infection. This example shows that co-inhabitants of a giv932 en microbiome can act as a source of genes for one another, particularly in cases of phagocytic 933 species like T. vaginalis [99].

934 The human microbiome has been referred to as "a hotspot of microbial horizontal gene 935 transfer" [100]. While studies on LGT in the human microbiome has almost entirely focused on 936 the extensive prokaryote-prokaryote transfers (e.g., 13,514 high confidence LGTs between 308 937 human microbiome bacteria [100]), diverse eukaryotes with lifestyles ranging from parasitic to 938 beneficial also reside in and are important components of the human microbiome [101]. The 939 abundance of a variety of species and phages in close proximity presumably creates a favorable 940 environment for LGT for microbial eukaryotes; by increasing the frequency of donor DNA in the 941 environment you increase the exposure of a species to a given DNA source $[13,44,64]$. 
942 Figure 1. Possible sources of foreign DNA contributing to LGT in eukaryotes. Eukaryotic

943 cells are exposed to many potential sources of exogenous DNA including from: i) lysed

944 endosymbiont-derived organelles (mitochondria and chloroplasts), prokaryotic or eukaryotic

945 endosymbionts, ii) viral infection, iii) bacterial conjugation (e.g., Agrobacterium tumefaciens)

946 and iv) lysed phagosomes containing food organisms.

947

948 Figure 2. The pan-genome concept. The pan-genome consists of all genes found in all strains

949 of a given species and is depicted at the top of the figure as the union of all ellipses (each ellipse

950 is the set of genes present in that strain) in the Venn diagram. The pan-genome can be further

951 broken down into the 'core' genome (genes found in all strains shown in dark teal) and the 'ac-

952 cessory' genome (shown colours ranging from green to blue) which are genes found in a single

953 strain (i.e. strain specific) or a subset of strains (i.e. strain select). 